\title{
Black holes on thick branes
}

\author{
Roberto Emparan \\ Departamento de Física Teórica, Universidad del País Vasco, Apdo. 644, E-48080 Bilbao, Spain \\ Ruth Gregory \\ Centre for Particle Theory, Durham University, South Road, Durham DH1 3LE, United Kingdom \\ Caroline Santos \\ Centre for Particle Theory, Durham University, South Road, Durham DH1 3LE, United Kingdom \\ and Departamento de Física da Faculdade de Ciências da Universidade do Porto, Rua do Campo Alegre 687, 4150 Porto, Portugal
}

(Received 10 January 2001; published 26 April 2001)

\begin{abstract}
The interplay between topological defects (branes) and black holes has been a subject of recent study, motivated in part by interest in brane-world scenarios. In this paper we analyze in detail the description of a black hole bound to a domain wall (a two-brane in four dimensions), for which an exact description in the limit of zero wall thickness has been given recently. We show how to smooth this singular solution with a thick domain wall. We also show that charged extremal black holes of a size (roughly) smaller than the brane thickness expel the wall, thereby extending the phenomenon of flux expulsion. Finally, we analyze the process of black hole nucleation on a domain wall, and argue that it is preferred over a previously studied mechanism of black hole nucleation away from the wall.
\end{abstract}

DOI: 10.1103/PhysRevD.63.104022

PACS number(s): 04.40.-b, 04.70.Bw, 04.70.Dy, 11.27.+d

\section{INTRODUCTION}

The study of the interaction of topologically nontrivial field theoretic solutions and black holes in four dimensions has yielded some interesting insights in recent years, revising our understanding of the classic "no-hair' theorems for black holes [1], with the realization that black holes can have "dressed" horizons [2], or even topological hair, in the guise of cosmic strings, extending to infinity [3]. An important feature of these (and other, related, solutions in the literature) is that they explicitly include gravitational back reaction of the defect on the black hole spacetime. For a localized defect, this is clearly a dressed version of a Reissner-Nordstrom (RN) black hole, however, for an extended defect, such as the cosmic string, the significant global aspects of the spacetime solution must be taken into account before claims of black hole hair can be validated. Fortunately, the spacetime of an infinitesimally thin string with a black hole is known: the Aryal-Ford-Vilenkin (AFV) [4] solution for a thin string piercing a black hole (a RN black hole with a wedge cut out), the Israel-Kahn metric [5] for two black holes suspended in unstable equilibrium by two strings ending on the event horizons, and the $C$ metric [6] corresponding to a black hole being accelerated by a string extending to infinity. All of these metrics can have their conical deficits smoothed by a realistic vortex core model $[3,7]$.

Also in recent times, domain walls (and other defects) have become a subject of intense study from the point of view that our universe might be a brane, or defect (see [8] for pioneering work), sitting in some higher dimensional spacetime. The motivation has come partly because string or $\mathrm{M}$ theory appears to admit a phase in which our world appears as a "wall" [9], but also because of the exciting phenomenological possibility of an unusual resolution of the hierarchy problem [10]. Most attention has been focused on the case where our universe is a gravitating domain wall $[11,12]$, however, higher codimension compactifications have been considered [13]. A general feature of these solutions is that four-dimensional gravity is recovered on the brane universe $[12,14]$, at least perturbatively, although the question of nonlinear effects, such as black holes on the brane [15], remains an interesting open one, necessitating a study of the problem in one dimension less [16]: i.e., a black hole living on a wall in four spacetime dimensions.

A natural question, when considering our universe as a brane, is to investigate models in which our universe is, quite literally, a defect, namely, a "soliton" solution to some higher dimensional field theory. This approach was taken in [13], and for the domain wall in five dimensional anti-de Sitter space, by Gremm [17]. It appears that one can smooth out the "singular"' wall by modeling it with the core of a topological domain wall. In a similar fashion to the infinitesimally thin brane, one can ask questions about strong gravity on the thick brane, namely a black hole intersecting a thick brane. This is the question we are interested in in this paper, and for the same reason as the infinitesimally thin wall, we will examine this issue in four-dimensional gravity.

Recently, it was shown numerically that a topological domain wall could sit through a Schwarzschild black hole [18] in the absence of gravitational back reaction. In the light of the results for the vortex solution, [3], this is perhaps not surprising, however, the issue of gravitational back reaction is particularly important in this setup. The gravitational field of a domain wall was found some time ago [19], and in a coordinate system natural to the wall (i.e., exhibiting planar symmetry) was found to be time-dependent-in stark contrast to the static conical metric of the cosmic string-with a de Sitter like expansion along the spatial coordinates of the wall. Later, as the global structure of the wall spacetime was better understood, it was realized that the horizon singulari- 
ties of the wall spacetime were removable by transforming into a "bulk-based" coordinate system, in which spacetime is flat, and consists of the interior of two hyperboloids in Minkowski spacetime glued together [20]. Space is compact in the domain wall spacetime, and the horizon a consequence of the acceleration of the bubble. Placing a black hole on the wall therefore involves placing a black hole on this accelerating wall with its compact space. An additional question is how charge on the black hole affects the domain wall. At first sight, one might think that using a Reissner-Nordström black hole, rather than Schwarzschild, for the background field theory solution should make no difference, however, this ignores the issue of flux expulsion. For an extreme black hole, there is a phenomenon of flux expulsion [21-23] in which if the vortex is thick enough, the black hole will expel its flux, causing the field to remain in its symmetric state on the horizon. Such a flux expulsion is not dependent on the vortex being local, it occurs for global strings [23] and for pure flux p-branes [22], therefore we might well expect a similar phenomenon to occur for the domain wall. All of this discussion however, hinges on not only the existence of a suitable thin wall metric with a black hole, but on this being a thin wall limit of a smooth thick wall metric with a black hole sitting on it. Note that unlike the vortex, the strong gravitational effect of the domain wall will mean that as soon as we include gravitational back reaction, the whole nature and global structure of the spacetime will change. Indeed, even in the absence of a black hole, there is a closely related phenomenon of wall non-formation: if the thickness of the wall is too great compared to the inverse mass of the scalar field forming it, then it is not possible to form a domain wall $[24,25]$.

This paper addresses the issues raised above. Fortunately, the metric of an infinitesimally thin wall has been found in [16] for the case of a wall in AdS spacetime, by using the $C$ metric for the accelerating wall, sliced in two and identified. In this paper, we show how to smooth such singular solutions with a thick domain wall thereby demonstrating the smooth wall + black hole spacetime. We will work with the solutions where the cosmological constant in the bulk of spacetime vanishes, but extrapolation to a nonzero cosmological constant should be straightforward. As expected, the space is compact, and the black holes are accelerated along with the wall. We also explore the question of "flux" expulsion for the wall, proving analytically that such expulsion occurs, and deriving bounds for the mass of the black hole (in wall units) at which it must occur. We then analyze the process of black hole nucleation on a domain wall. Unlike the decay of strings [7], the net result of this process is not the disintegration of the wall, but rather a pair creation of black holes in the presence of the accelerating wall.

The layout of the paper is as follows: In the next section we review and extend the work of [16], deriving an appropriate infinitesimal wall + black hole metric and analyzing its thermodynamics. In Sec. III we consider the field equations of the domain wall in the background of a black hole (both RN and the $C$ metric), deriving an analytic "thinwall" approximation which will be useful for the problem of gravitational back reaction, and then demonstrating, in Sec.
IV, the phenomenon of flux expulsion. Section V deals with gravitational back reaction, and Sec. VI with the nucleation of black holes on the wall. Finally, we summarize our results in Sec. VII, and discuss the possible consequences and extensions to the scenario with a three-brane in five dimensions, of relevance to brane world models.

\section{THE BLACK HOLE-WALL METRIC}

\section{A. Constructing the solution}

We start by deriving the equivalent of the AFV solution for the vortex, namely an infinitesimally thin domain wall with a black hole. We begin with the $C$ metric [6]

$$
d s^{2}=\frac{1}{A^{2}(x+y)^{2}}\left[F(y) d t^{2}-\frac{d y^{2}}{F(y)}-G(x) d \varphi^{2}-\frac{d x^{2}}{G(x)}\right],
$$

where $G(x)=1-x^{2}-2 m A x^{3}-q^{2} A^{2} x^{4}=-F(-x)$. A bulk cosmological constant $\Lambda$ could be easily incorporated, and in particular a negative one may be of interest to discuss toy models for the Randall-Sundrum scenario (see [16]). For simplicity, we set $\Lambda=0$ : the results in Secs. III, IV and V can be easily extended to non-zero $\Lambda$. On the other hand, issues such as global structure, thermodynamics and instantons as studied here, extend qualitatively to all cases where the geometry induced on the brane is de Sitter, even if $\Lambda$ is positive or negative.

In general the quartic $G(\xi)$ will have 4 roots, and we will take the parameters such that they are all real, and labeled as $\xi_{1} \leqslant \xi_{2}<\xi_{3}<0<\xi_{4} \leqslant 1$. The coordinates in Eq. (1) are restricted to $x \in\left[\xi_{3}, \xi_{4}\right]$, and $y \in(-x, \infty) ; y=-\xi_{3},-\xi_{2}$, $-\xi_{1}(>0)$ are the acceleration horizon, and outer and inner black hole horizons, respectively. For $m \neq 0$ there is a singularity at $y=\infty$, which corresponds to the central singularity of the black hole. Note also that in general this spacetime has a conical deficit as $x \rightarrow \xi_{3}, \xi_{4}$, one of which (say at $\xi_{4}$ ) can be eliminated by setting the periodicity of $\varphi$ to be $\varphi$ $\in\left[0,4 \pi /\left|G^{\prime}\left(\xi_{4}\right)\right|\right]$. The remaining conical deficit at $x=\xi_{3}$ has the interpretation of a string pulling the accelerating black hole away to infinity. The gauge potential for the Maxwell field is $\mathbf{A}=q y \mathbf{d} t$ for an electric black hole, or $\mathbf{A}=q(x$ $\left.-\xi_{4}\right) \mathbf{d} \varphi$ for a magnetic black hole.

The conventional definitions of mass [say, ArnowittDeser-Misner (ADM)] cannot be applied to obtain the mass of the accelerating black hole, ${ }^{1}$ however, for a small black hole, i.e., $m, q \ll 1 / A$, the geometry approaches the ReissnerNordstrom solution, which allows us to identify approximately the black hole mass as $m / G$. Indeed, it is useful to have the approximate values for the roots for a small black hole:

\footnotetext{
${ }^{1}$ See, however, below.
} 


$$
\begin{gathered}
\xi_{1}=-\frac{1}{r_{-} A}+O(m A), \quad \xi_{2}=-\frac{1}{r_{+} A}+O(m A), \\
\xi_{3}=-1-m A+O\left(m^{2} A^{2}\right), \quad \xi_{4}=1-m A+O\left(m^{2} A^{2}\right),
\end{gathered}
$$

with $r_{ \pm}=m \pm \sqrt{m^{2}-q^{2}}$ (we are taking $m \geqslant q$; the extremality bound is precisely $\xi_{2} \geqslant \xi_{1}$ ). The charge of the black hole can be measured by integrating the flux on a sphere that surrounds it (e.g., at $y=$ const), and is given by

$$
\begin{aligned}
Q & =\frac{1}{4 \pi} \int F_{x \varphi} d x d \varphi=\frac{\Delta \varphi}{4 \pi}\left[A_{\varphi}\left(x=\xi_{4}\right)-A_{\varphi}\left(x=\xi_{3}\right)\right] \\
& =\frac{1}{q A^{2}} \frac{1}{\left(\xi_{4}-\xi_{2}\right)\left(\xi_{4}-\xi_{1}\right)} \\
& =q+O(m A),
\end{aligned}
$$

where $\Delta \varphi=4 \pi /\left|G^{\prime}\left(\xi_{4}\right)\right|$ is the period of $\varphi$.

To construct the wall-black-hole (WBH) metric we follow the standard Israel construction, according to which the tension $\sigma$ of a domain wall is given by the discontinuity of the extrinsic curvature, $\left[K_{i j}\right]=4 \pi G \sigma h_{i j}$, with $h_{i j}$ the metric induced on the wall. Following [16], an appropriate totally umbilic surface $\left(K_{i j} \propto h_{i j}\right)$ can be found at $x=0$. This has normal $n=(1 / A y) d x$, induced metric

$$
d s^{2}=\frac{1}{A^{2} y^{2}}\left[F(y) d t^{2}-\frac{d y^{2}}{F(y)}-d \varphi^{2}\right],
$$

and extrinsic curvature

$$
K_{i j}=A h_{i j} .
$$

We have chosen the conical singularity to lie at $x=\xi_{3}$, i.e., on the side $x<0$ of this surface. As a consequence, if we form the WBH solution by taking two copies of the side $x$ $>0$ and glue them together along $x=0$, the string will have disappeared from the spacetime. The construction is equivalent to substituting $|x|$ for $x$ in Eq. (1). Note however, that the gauge potential remains unaltered. This is evident for the case of an electric potential (which does not depend on $x$ ), while for a magnetic potential the change in sign in $x$ is canceled by a corresponding reversal of $\mathbf{d} \varphi$. The Israel construction implies that the tension of the wall thus formed is

$$
\sigma=\frac{A}{2 \pi G} .
$$

On the other hand, the charge of the black hole changes now to

$$
\begin{aligned}
Q & =2 \frac{\Delta \varphi}{4 \pi}\left[A_{\varphi}\left(x=\xi_{4}\right)-A_{\varphi}(x=0)\right] \\
& =\frac{2}{q A^{2}} \frac{\xi_{4}}{\left(\xi_{4}-\xi_{3}\right)\left(\xi_{4}-\xi_{2}\right)\left(\xi_{4}-\xi_{1}\right)},
\end{aligned}
$$

but, to leading order in $m A$, we still have $Q \simeq q$.
The metric induced on the wall takes a particularly interesting form if we introduce a radial coordinate $r=1 /(A y)$, and $t=A \bar{t}$. Then, Eq. (4) becomes

$$
\begin{aligned}
d s^{2}= & \left(1-\frac{2 m}{r}+\frac{q^{2}}{r^{2}}-A^{2} r^{2}\right) d \bar{t}^{2} \\
& -\frac{d r^{2}}{1-\frac{2 m}{r}+\frac{q^{2}}{r^{2}}-A^{2} r^{2}}-r^{2} d \varphi^{2}
\end{aligned}
$$

which is exactly the same as the equatorial section of the four dimensional Reissner-Nordström-de Sitter solution.

The domain wall that we have constructed actually contains two black holes, sitting at antipodal points of a spherical domain wall. In order to see this, let us have a closer look at the global structure of the WBH spacetime. It is helpful to consider first the situation where the black holes are absent from the wall, i.e., $q=m=0$. In that case, the metric is

$$
\begin{aligned}
d s^{2}= & \frac{1}{A^{2}(x+y)^{2}} \\
& \times\left[\left(y^{2}-1\right) d t^{2}-\frac{d y^{2}}{y^{2}-1}-\frac{d x^{2}}{1-x^{2}}-\left(1-x^{2}\right) d \varphi^{2}\right] .
\end{aligned}
$$

The acceleration horizon is at $y=+1$. If we now change to coordinates $(T, X, Y, Z)$, using

$$
\begin{aligned}
X^{2}-T^{2} & =\frac{y^{2}-1}{A^{2}(x+y)^{2}}, \quad Y^{2}+Z^{2}=\frac{1-x^{2}}{A^{2}(x+y)^{2}} \\
\frac{T}{X} & =\tanh t, \quad \frac{Z}{Y}=\tan \varphi
\end{aligned}
$$

we find that we recover Minkowski space,

$$
d s^{2}=d T^{2}-d X^{2}-d Y^{2}-d Z^{2} .
$$

Since

$$
X^{2}+Y^{2}+Z^{2}-T^{2}=\frac{1}{A^{2}} \frac{y-x}{y+x},
$$

it follows that the surface at $x=0$ where the domain wall lies, corresponds in Minkowski space to the hyperboloid (see Fig. 1)

$$
X^{2}+Y^{2}+Z^{2}-T^{2}=\frac{1}{A^{2}}
$$

and the region $x>0$ is the interior of the hyperboloid $X^{2}$ $+Y^{2}+Z^{2}-T^{2}<1 / A^{2}$. Sections at constant $T$ are spheres, so the spatial geometry of the wall is spherical. In fact, the intrinsic geometry of the wall is precisely that of de Sitter space in $2+1$ dimensions, as is evident from Eq. (8).

Now we add the black holes, by allowing for $m>0$. In this case, $y=+\infty$ is a singularity, surrounded by at least one 


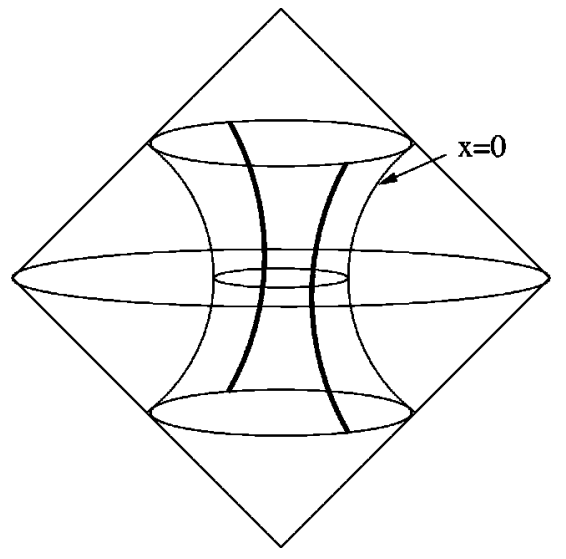

FIG. 1. Conformal diagram for the embedding of the hyperbolic surface $x=0$ in Minkowski spacetime (circles in this picture are actually spheres). The thick lines correspond to $y=+\infty$, which track the world lines of the black holes in the $C$ metric.

black hole horizon at $y=\left|\xi_{2}\right|$ (and an inner black hole horizon $y=\left|\xi_{1}\right|$ if $q \neq 0$ ). This implies that, if the black hole is not too large, we can get a good approximation for its position on the wall by looking at the trajectories of points at large positive values of $y$ in the metric (9). In particular, the central singularity of the black hole, at $y=+\infty$ is mapped to $Y^{2}+Z^{2}=0, \quad X^{2}-T^{2}=1 / A^{2}$, i.e., $X= \pm \sqrt{T^{2}+1 / A^{2}}, \quad Y=Z$ $=0$. At any given instant $T$, these are $t w o$ points at antipodal points on the domain wall. Therefore, the WBH metric actually describes two black holes at antipodal points of a spherical domain wall. It is easy to see that the black holes must be oppositely charged.

Note that the domain wall not only eliminates the string from the spacetime, but it also cuts off the acceleration horizon and turns it into a horizon of finite area. The entire construction is depicted in Fig. 2.

Finally, another interesting point is that, as argued in [26], the black holes will neither swallow up the brane, nor slide off of it. The latter was argued on the basis of the elastic restoring force that the brane exerts on the black hole. Below we will give an alternative, thermodynamic argument for this feature.

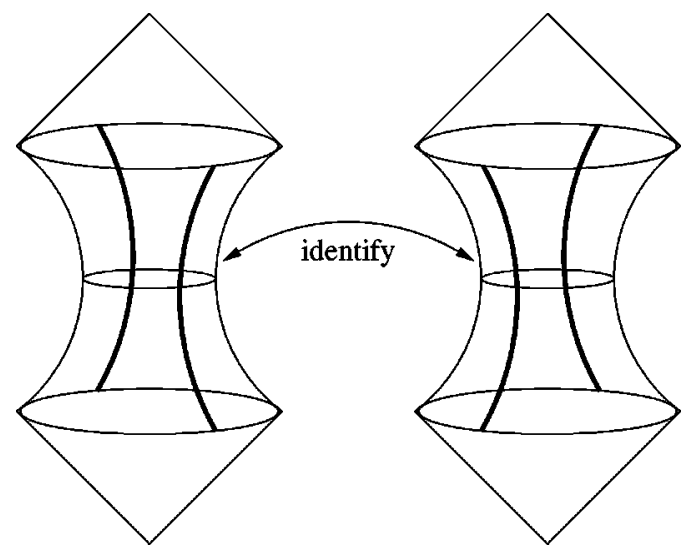

FIG. 2. Construction of the domain wall by gluing two copies of the region $x>0$ along the surface $x=0$. The wall tension results from the non-vanishing extrinsic curvature of this surface.

\section{B. Thermodynamics of a black hole on the wall}

The conventional definitions for the mass of the black hole, e.g., ADM, cannot be applied to a spacetime such as the present one, which is not asymptotically flat. Nevertheless, black hole thermodynamics can be used to give a prescription for the mass. This approach was put to use in [16], where it was shown to yield very satisfactory results. The method consists of identifying the black hole entropy using the area formula $S=\mathcal{A}_{\mathrm{bh}} / 4 G$, and the temperature $T$ in terms of the surface gravity $\kappa, T=\kappa / 2 \pi$. Then, the first law of thermodynamics (in the absence of charge),

$$
\delta M=T \delta S
$$

can be integrated to give the black hole mass $M$. For simplicity, we choose to set to zero the black hole charge in this subsection. This means that $G(x)=1-x^{2}-2 m A x^{3}$, and $\xi_{1}$ $\rightarrow-\infty$, but we remain consistent with the notation so far, i.e., $y=\left|\xi_{2}\right|$ corresponds to the location of the black hole horizon, $0 \leqslant x \leqslant \xi_{4}$, etc. ${ }^{2}$

The calculation of the area is straightforward,

$$
\begin{aligned}
\mathcal{A}_{\mathrm{bh}} & =\left.\int d x d \varphi \sqrt{g_{x x} g_{\varphi \varphi}}\right|_{y=\left|\xi_{2}\right|}=2 \frac{\Delta \varphi}{A^{2}} \int_{0}^{\xi_{4}} \frac{d x}{\left(x+\left|\xi_{2}\right|\right)^{2}} \\
& =2 \frac{\Delta \varphi}{A^{2}} \frac{\xi_{4}}{\left|\xi_{2}\right|\left(\left|\xi_{2}\right|+\xi_{4}\right)} .
\end{aligned}
$$

The factor of 2 in the second line arises from the doublesided character of the wall.

The surface gravity is computed relative to the timelike Killing vector $\partial_{t}$. In order for it to have the right dimensionality, we have to multiply it by a factor of $A, \chi=A \partial_{t}$, and then $\kappa^{2}=\chi_{\mu ; \nu} \chi^{\mu ; \nu} / 2$. With this normalization for $\chi$ one also recovers the standard result for the temperature of the Schwarzschild-de Sitter black hole on the brane (8). The result is

$$
T=\frac{A\left|F^{\prime}\left(-\xi_{2}\right)\right|}{4 \pi}=\frac{A\left|G^{\prime}\left(\xi_{2}\right)\right|}{4 \pi} .
$$

In order to perform the integration of the first law it is convenient to introduce the auxiliary variable

$$
z=\frac{\left|\xi_{2}\right|}{\xi_{4}}
$$

The limit where the black holes are absent, $m=0$, corresponds to $z \rightarrow \infty$. On the other hand, there is a lower bound for $z$ imposed by the fact that $G(x)$ must have three real roots. This requires $m A<1 / 3 \sqrt{3}$, hence $\left|\xi_{2}\right|>\sqrt{3}$ and $z>2$ (when this bound is saturated the black hole and acceleration horizons coincide). Therefore the range for $z$ is $2<z<\infty$.

\footnotetext{
${ }^{2}$ Notice that here we are out of thermodynamic equilibrium, in the sense that the temperature of the black hole and acceleration horizons are different. This poses no problem at this point, but see Sec. VI for more on this.
} 
In terms of $z$ we have

$$
\begin{aligned}
\xi_{4} & =\frac{\sqrt{z^{2}-z+1}}{z}, \quad\left|\xi_{2}\right|=\sqrt{z^{2}-z+1}, \\
m A & =\frac{z(z-1)}{2\left(z^{2}-z+1\right)^{3 / 2}},
\end{aligned}
$$

and

$$
\begin{aligned}
& S=\frac{2 \pi}{G A^{2}} \frac{z}{(1+z)^{2}(2 z-1)}, \\
& T=\frac{A}{4 \pi} \frac{z^{2}-z-2}{\sqrt{z^{2}-z+1}} .
\end{aligned}
$$

In the range $2<z<\infty, m$ and $S$ decrease monotonically with $z$, while $T$ grows monotonically with it. The first law

$$
\frac{d M}{d z}=T \frac{d S}{d z}
$$

can now be integrated, with the condition that $M=0$ for $m$ $=0$, i.e., for $z \rightarrow \infty$. In this way we find that the black hole mass is

$$
M=\frac{1}{G A}\left(\frac{1}{2}-z \frac{\sqrt{z^{2}-z+1}}{2 z^{2}+z-1}\right) .
$$

Let us examine what these formulas yield for small black holes, up to next to leading order in $m A$. Using Eq. (2),

$$
\begin{aligned}
M & =\frac{m}{G}\left(1-\frac{15}{4} m A+O\left(m^{2} A^{2}\right)\right), \\
S & =\frac{4 \pi m^{2}}{G}\left(1-5 m A+O\left(m^{2} A^{2}\right)\right) .
\end{aligned}
$$

If we now express the entropy in terms of the physical mass $M$, and the wall tension $\sigma$,

$$
S=4 \pi G M^{2}\left(1+5 \pi G^{2} \sigma M+O\left(G^{4} M^{2} \sigma^{2}\right)\right) .
$$

The leading order result reproduces the standard formula for Schwarzschild black holes. The first correction tells us that, for a given black hole mass, the entropy will be higher if it is on a wall, than if it is away from it. This feature persists throughout the entire range of masses, as exhibited in Fig. 3. Hence, it is thermodynamically favored for a black hole to stick to the wall, in accord with the above mentioned fact that black holes do not slide off of the wall.

We end this analysis with two remarks related to the existence of an upper limit for the size of the black holes on the wall. First, one may notice that $z$ can be allowed a range wider than considered above, namely, one can also have 1 $\leqslant z \leqslant 2$ without encountering any singular behavior. However, while the limit $z \rightarrow 2$ from above corresponds to $m A$ $\rightarrow 1 / 3 \sqrt{3}$ from below, going to $1 \leqslant z<2$ does not correspond to having larger $m A$ (since we still have three real roots), but

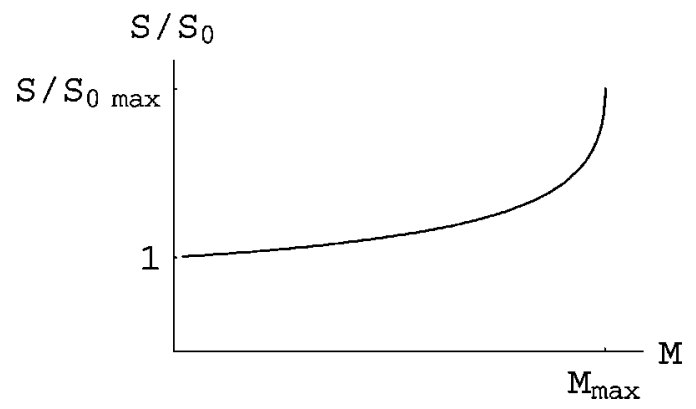

FIG. 3. The entropy of a black hole on the wall, $S$, compared to the entropy of a black hole away from the wall, $S_{0}=4 \pi G M^{2}$. Notice $S>S_{0}$ for all allowed values of $M$. The maximum values correspond to $z \rightarrow 2$.

rather to exchanging $\xi_{2}$ and $\xi_{3}$, i.e., the black hole and acceleration horizons. For $1 \leqslant z \leqslant 2$, the plot in Fig. 3 would extend to yield the entropy (area) of the acceleration horizon, for a given black hole mass.

The second remark concerns an aspect of the shape of black holes on branes discussed in Ref. [16]. There, it was argued that large black holes in the four dimensional RS brane-world have the shape of a pancake, the horizon being flattened and having a small extent away from the brane. Is there anything similar in the present context? It would appear that there is not, at least not anything as drastic as in [16]. The crucial difference is that in the situation studied in [16], the geometry of the brane was not asymptotically de Sitter, but instead, asymptotically flat. The "black pancakes" corresponded to $m A$ becoming larger than $1 / 3 \sqrt{3}$. We have explicitly excluded this from our analysis: the reason is that for $m A=1 / 3 \sqrt{3}$ the black hole horizon and the acceleration horizon (which yields the cosmological de Sitter horizon on the brane) become coincident, and for $m A>1 / 3 \sqrt{3}$ there is no black hole on the brane, but rather a naked singularity. This obstruction obviously disappears for the asymptotically flat brane of [16], and in that case the horizon on the brane can grow arbitrarily large. There are no "black pancakes" in our setting, and all our black holes are roughly spherical. On the other hand, if there were a negative cosmological constant in the bulk, but a positive cosmological constant induced on the brane, the cosmological brane horizon would be larger than in the present setting, and the upper limit on the size of the black holes would be higher.

\section{THIN WALL APPROXIMATION}

Having described in detail the wall-black hole spacetime in the limit of infinitesimal wall thickness, we now proceed to demonstrate how the distributional wall, with the black hole on it, can consistently arise as a limit of a physical, field-theoretical topological defect. This is needed to establish the analytic approximation which will be used to derive the gravitational back reaction of the wall on the spacetime.

We will use a general field theory Lagrangian for the wall:

$$
8 \pi G \mathcal{L}_{\mathrm{DW}}=\frac{\epsilon}{w^{2}}\left[w^{2}\left(\nabla_{a} X\right)^{2}-V(X)\right]
$$


where the symmetry breaking potential $V$ has a discrete set of degenerate minima, and we have rescaled the scalar field $X(=\Phi / \eta)$ by the symmetry breaking parameter $\eta$. The parameter $\epsilon=8 \pi G \eta^{2}$ represents the gravitational strength of the domain wall, being the energy density per unit area, and $w$ represents the inverse mass of the scalar after symmetry breaking, which will also characterize the width of the wall defect within the theory. Without loss of generality, we will fix our units by setting $w=1$. The wall equations

$$
\square X+\frac{1}{2} \frac{\partial V}{\partial X}=0
$$

have the first integral $X^{\prime 2}=V(X)$ in Minkowski spacetime, which has an implicit solution

$$
\int_{X_{F}}^{X} \frac{d X}{\sqrt{V(X)}}=z-z_{0}
$$

where $X_{F}=X\left(z_{0}\right)$ is the false vacuum. For example, in the $\lambda \Phi^{4}$ kink model, $w=1 / \sqrt{\lambda} \eta=1$, and the above integral (26) gives the usual kink solution centered on $z_{0}: X=\tanh (z$ $-z_{0}$ ), which has an energy per unit area

$$
8 \pi G \sigma=2 \epsilon \int_{-\infty}^{\infty} \operatorname{sech}^{4} z=\frac{8 \epsilon}{3} .
$$

Now let us consider the wall equations (25) in the Reissner-Nordström background

$$
\begin{aligned}
d s^{2}= & \left(1-\frac{2 M}{r}+\frac{Q^{2}}{r^{2}}\right) d t^{2}-\left(1-\frac{2 M}{r}+\frac{Q^{2}}{r^{2}}\right)^{-1} \\
& \times d r^{2}-r^{2} d \theta^{2}-r^{2} \sin ^{2} \theta d \varphi^{2}
\end{aligned}
$$

where $M$, and $Q$ are measured in "wall" units, (i.e., $w=1$ ) rather than Planck units, and the numerical value of $G$ in wall units has been absorbed into $M$ and $Q$. This gives for the scalar $X$ :

$$
\begin{gathered}
\left(1-\frac{2 M}{r}+\frac{Q^{2}}{r^{2}}\right) X_{, r r}+\frac{2}{r}\left(1-\frac{M}{r}\right) X_{, r} \\
+\frac{1}{r^{2}} X_{, \theta \theta}+\frac{\cot \theta}{r^{2}} X_{, \theta}=\frac{1}{2} \frac{\partial V}{\partial X} .
\end{gathered}
$$

Recall that for the case of the vortex, [3], the fields were very well approximated as functions of $r \sin \theta$, therefore, guessing the Ansatz $X=X(\mathbf{z})=X(r \cos \theta)$, we find that

$$
\square X=-X^{\prime \prime}\left[1-\frac{2 M \mathbf{z}^{2}}{r^{3}}+\frac{Q^{2} \mathbf{z}^{2}}{r^{4}}\right]+\frac{2 M \mathbf{z}}{r^{3}} X^{\prime} .
$$

Now, noting that $r$ is strictly greater than $M$ outside the horizon of an RN black hole, we see that the $\mathbf{z}$-dependent terms in Eq. (30) are of order $\mathbf{z} M^{-2}$ or $\mathbf{z}^{2} M^{-2}$. Therefore, if the thickness of the wall is much less than the black hole horizon size, i.e., $M \gg 1$, we see that $X=X_{0}(\mathbf{z})$, where $X_{0}$ is the flat space solution of Eq. (26), will solve the equations of motion up to $\mathrm{O}\left(M^{-2}\right)$, since the derivatives of $X_{0}$ differ from zero significantly only for $\mathbf{z}=\mathrm{O}(1)$. Thus we see that a thin wall can be painted on to a black hole solution, as confirmed by the numerical work in [18].

However, since we expect our gravitating wall-black hole system to have a metric of the form (1), we have to see how to paint the wall onto the $C$ metric, which at first sight is a rather different looking beast, however, if we change coordinates via

$$
\bar{t}=A^{-1} t, \quad r=1 / A y, \quad \text { and } \quad \theta=\int_{x}^{x_{3}} d x / \sqrt{G(x)}
$$

then

$$
\begin{aligned}
d s^{2}= & {[1+\operatorname{Arx}(\theta)]^{-2}\left[\left(1-\frac{2 m}{r}+\frac{q^{2}}{r^{2}}-A^{2} r^{2}\right) d \bar{t}^{2}\right.} \\
& \left.-\frac{d r^{2}}{\left(1-\frac{2 m}{r}+\frac{q^{2}}{r^{2}}-A^{2} r^{2}\right)}-r^{2} d \theta^{2}-r^{2} G(x) d \varphi^{2}\right]
\end{aligned}
$$

We see therefore that the variable $x$ is basically $\cos \theta$, and therefore we guess that $\mathbf{z}=x / A y$. Substituting this into the wave operator for $X$ gives

$$
\begin{aligned}
-\square X= & X^{\prime \prime}(1+A \mathbf{z})^{2}\left[G(x)+A^{2} \mathbf{z}^{2} F(y)\right]+X^{\prime} A(1+A \mathbf{z}) \\
& \times\left[2 G(x)+2 A \mathbf{z} F(y)(2+A \mathbf{z})+\left(y G^{\prime}(x)\right.\right. \\
& \left.\left.-x F^{\prime}(y)\right)(1+A \mathbf{z})\right] .
\end{aligned}
$$

We now need to consider what the "thin wall" approximation means in the context of the $C$ metric. Clearly we expect the black hole horizon radius to be large in these units, i.e., $A\left|\xi_{2}\right| \ll 1$, however, recall that for a self-gravitating domain wall, there is a limit to wall formation given by the size of the spontaneously compactified spacetime, which corresponds to the acceleration horizon. Therefore, although we are not at this point considering gravitational back reaction, we will also work in the régime of large acceleration radius, i.e., $A\left|\xi_{3}\right| \ll 1$. Now, the wall fields differ significantly from their vacuum values for $\mathbf{z} \sim 1$, and the values of $y$ are bounded by the black hole horizon, and the acceleration horizon $1<\left|\xi_{3}\right| \leqslant y<\left|\xi_{2}\right|$. Therefore $x \leqslant A y \ll 1$ in the core of the wall. Meanwhile, the maximum value of $F(y)$ is at most of order $\left|\xi_{2}\right|^{2}$, hence we see that

$$
-\square X=X^{\prime \prime}+O(A)
$$

So $X=X_{0}(\mathbf{z})$ is indeed a good approximation to the solution of the field equation in the $C$ metric background.

\section{EXTREMAL HORIZONS EXPEL THICK WALLS}

Having argued the existence of the domain wall solution in the black hole background for large mass black holes, we will now consider the special case of an extremal black hole, 
for which the inner and outer horizons coincide. First consider the extremal RN black hole

$d s^{2}=\left(1-\frac{M}{r}\right)^{2} d t^{2}-\left(1-\frac{M}{r}\right)^{-2} d r^{2}-r^{2} d \theta^{2}-r^{2} \sin ^{2} \theta d \varphi^{2}$.

For the cosmic string, or a pure flux p-brane, a phenomenon of "flux expulsion", occurs for sufficiently small mass black holes, namely, the defect ceases to penetrate the black hole horizon, and instead wraps around it with the horizon remaining in the symmetric phase across all of its area. If such a phenomenon occurs with the domain wall, this would mean that $X \equiv 0$ over the event horizon, and all of the nontrivial field dynamics of the wall would occur in the exterior region. Although the above thin wall approximation indicates that for large mass black holes there is a solution with the wall intersecting the black hole, it gives no indication of what might happen for $M \leqslant O(1)$.

Indeed, a simple argument gives us a first indication that at least very small extremal black holes sitting well inside the wall will expel it. Deep inside the core of the wall the potential terms are very small compared to the gradient terms, so we neglect them. In this approximation, we now try to solve the wall equations in the RN background, i.e., Eq. (29) with the RHS set to zero. Given that, in the absence of a black hole, the solution for a wall in the region close to its center is $X \approx \mathbf{z}-\mathbf{z}_{0}$, we try the ansatz $X=b(r) \cos \theta$, and obtain the equation

$$
\left(r^{2}-2 M r+Q^{2}\right) b^{\prime \prime}+2(r-M) b^{\prime}-2 b=0,
$$

which admits $b=r-M$ as the regular solution at the horizon, i.e.,

$$
X \approx(r-M) \cos \theta \text {. }
$$

We see that if the black hole is not extremal, then $X \neq 0$ on the horizon, but $X$ will vanish on an extremal horizon. We now derive a more precise and rigorous bound on $M$ for which the wall must be expelled from the black hole.

Suppose there is a solution to the equations of motion which penetrates the horizon, then on the horizon the field equations become

$$
X_{, \theta \theta}=-\cot \theta X_{, \theta}+2 M^{2} X\left(X^{2}-1\right),
$$

i.e., an ODE for $X$ in terms of $\theta$. Taking the derivative gives

$$
X_{, \theta \theta \theta}=-\cot \theta X_{, \theta \theta}+X_{, \theta}\left[\csc ^{2} \theta+2 M^{2}\left(3 X^{2}-1\right)\right] .
$$

Now, any nontrivial solution will satisfy $X(\pi / 2)=0$ which implies that $X_{, \theta \theta}(\pi / 2)=0$. Without loss of generality we may suppose that $X_{, \theta}(\pi / 2)>0$, so that $X_{, \theta}$ has a maximum (minimum) at $\pi / 2$ for $M^{2}>1 / 2\left(M^{2}<1 / 2\right)$ since Eq. (39) implies that at any turning point of $X_{, \theta}$

$$
X_{, \theta \theta \theta}=X_{, \theta}\left[\csc ^{2} \theta+2 M^{2}\left(3 X^{2}-1\right)\right]>X_{, \theta}\left[1-2 M^{2}\right]
$$

(for $X_{, \theta}>0$ ). But we now see that if $M^{2}<1 / 2$, any turning point of $X_{, \theta}$ is a minimum, which is inconsistent with $X_{, \theta}$ $=0$ at $\theta=0, \pi$. Therefore for $M^{2}<1 / 2$, the only possible solution is $X \equiv 0$ on the horizon, i.e., expulsion must occur. By continuity, we in fact expect the true limit on $M^{2}$ for expulsion to be somewhat higher than $1 / 2$, although numerical work would be required to establish the true bound. For the $C$ metric, the argument is slightly more involved, however, using the $\theta$-coordinate as defined in Eq. (31) we can derive a similarly weak bound for expulsion as $A\left|\xi_{2}\right|>1$.

Now let us examine the region $M^{2}>1 / 2$. If a stable expelling solution exists, we expect that in the region near the horizon, it monotonically relaxes to a kink solution as we move away from the horizon, namely, $X_{{ }_{\theta}}$ remains of the same sign (without loss of generality we will take $X_{, \theta}>0$ for $r>M)$. Let us consider the full field equations in a neighborhood of the horizon such that $r<M+\delta / M$ and $|X|<\delta$ for some small parameter $\delta$ :

$$
X_{, \theta \theta}=-\cot \theta X_{, \theta}-2 M^{2} X-\left[(r-M)^{2} X_{, r}\right]_{, r}+O(\delta) .
$$

By considering $\theta$ derivatives of this equation, it is possible to show that $X_{, \theta \theta}, X_{, \theta \theta \theta} \leqslant 0$ and $X_{, \theta \theta \theta \theta} \geqslant 0$ on $[\pi / 2, \pi]$ for $M^{2}$ $>1 / 2$; hence,

$$
\frac{\pi}{4} X_{, \theta}\left(\frac{\pi}{2}\right)<X(\pi)<\frac{\pi}{2} X_{, \theta}\left(\frac{\pi}{2}\right)
$$

and

$$
\frac{\pi}{4}\left|X_{, \theta \theta}(\pi)\right|<X_{, \theta}\left(\frac{\pi}{2}\right)<\frac{\pi}{2}\left|X_{, \theta \theta}(\pi)\right|
$$

Combining these inequalities, and reading off $X_{, \theta \theta}(\pi)$ from Eq. (41) gives

$$
\frac{\pi^{2} M^{2}}{16} X(\pi)<X(\pi)<\frac{\pi^{2} M^{2}}{4} X(\pi),
$$

i.e., $2 / \pi<1 / \sqrt{2}<M<4 / \pi$. Therefore, if $M>4 / \pi$ there can be no such expelling solution.

\section{GRAVITATIONAL BACK REACTION}

First let us briefly recall the self-gravitating domain wall. In wall-based coordinates the metric can be written in the form

$$
d s^{2}=a^{2}(z)\left[d t^{2}-e^{2 k t}\left(d x^{2}+d y^{2}\right)\right]-d z^{2},
$$

where the function $A(z)$, and the constant $k$, can be determined analytically as a power series in $\epsilon$, the leading order values being

$$
\begin{aligned}
a(z) & =1-\epsilon \int_{0}^{z} d z^{\prime} \int_{0}^{z^{\prime}} d z^{\prime \prime} V\left(X_{0}\left(z^{\prime \prime}\right)\right) \\
& =1-\frac{\epsilon}{6}\left[4 \log \cosh z+\tanh ^{2} z\right],
\end{aligned}
$$




$$
k=\epsilon \int_{0}^{\infty} d z^{\prime} V\left(X_{0}\left(z^{\prime}\right)\right)=\frac{2 \epsilon}{3},
$$

the explicit forms being those for the $\lambda \Phi^{4}$ kink. Note that the change in extrinsic curvature from one side of the kink to the other is $\left[a^{\prime}\right]=-4 \epsilon / 3=-4 \pi G \sigma$ from Eq. (27) as required by the Israel junction conditions. Comparing this with Eq. (5) for the WBH solution, we see that we must have the acceleration parameter $A=2 \epsilon / 3$ for consistency with the self-gravitating thick wall.

In general, our thick-WBH metric will take the form

$$
d s^{2}=\Omega^{-2}\left[E^{2} d t^{2}-B^{2} d y^{2}-D^{2} d x^{2}-C^{2} d \varphi^{2}\right],
$$

where to leading order $X=X_{0}$ and Eq. (47) takes the form of Eq. (1). We will now perform a linearized calculation in $\epsilon$ $=3 A / 2$, the energy of the wall, writing $\Omega=\Omega_{0}+A \Omega_{1}$ etc., where $\Omega_{1} / \Omega_{0}=O(1)$ near the core of the wall, and tends to zero away from the core.

Let us start by calculating the stress energy of the $X_{0}$-field:

$$
\begin{aligned}
g^{x x} X_{0, x} X_{0, x} & =-G(x) A^{2}(|x|+y)^{2}\left(\frac{X_{0}^{\prime}}{A y}\right)^{2} \simeq-X_{0}^{\prime 2}, \\
g^{y y} X_{0, y} X_{0, y} & =-F(y) A^{2}(|x|+y)^{2}\left(\frac{-\mathbf{z} X_{0}^{\prime}}{y}\right)^{2} \\
& =O\left(\left(A y_{h}\right)^{2}\right) .
\end{aligned}
$$

Since the gauge potential for the Maxwell field is unaltered by the presence of the wall, its energy momentum tensor remains formally the same:

$$
T_{\mathrm{EM}^{0}}^{0}=T_{\mathrm{EM}^{y}}^{y}=q^{2} \Omega^{4}=-T_{\mathrm{EM}^{x}}^{x}=-T_{\mathrm{EM}^{\varphi}}^{\varphi} .
$$

Therefore the Einstein equations for the wall,

$$
R_{a b}=2 \epsilon X_{, a} X_{, b}-\epsilon V(X) g_{a b}+T_{\mathrm{EM}^{a b}}
$$

become, to leading order in $A$,

$$
\begin{aligned}
R_{0}^{0}-q^{2} \Omega^{4} & =R_{\varphi}^{\varphi}+q^{2} \Omega^{4}=-\frac{3 A}{2} V(X) \\
& =R_{y}^{y}-q^{2} \Omega^{4}+O\left(A^{2}\right) \\
& =\frac{1}{3}\left(R_{x}^{x}+q^{2} \Omega^{4}\right)+O\left(A^{2}\right) .
\end{aligned}
$$

Since in Eq. (1) the variation of the extrinsic curvature due to the wall is primarily carried by $\Omega$, with $E_{0}^{2}=B_{0}^{2}=F(y)$ unaffected, and $C_{0}^{2}=D_{0}^{-2}=G(x)$ only affected at $O\left(x^{3}\right)$ $=O\left(A^{3} y^{3}\right)$ we guess that $E, B, C, D$ will effectively take their background values, in which case we find that the Ricci tensor is

$$
\begin{aligned}
R_{0}^{0}= & \frac{1}{2} \Omega^{2} F^{\prime \prime}(y)-2 F^{\prime}(y) \Omega \Omega_{, y}-G^{\prime}(x) \Omega_{, x}-[G(x) \\
& \left.\times\left(\Omega \Omega_{, x x}-3 \Omega_{, x}^{2}\right)+F(y)\left(\Omega \Omega_{, y y}-3 \Omega_{, y}^{2}\right)\right] \\
R_{\varphi}^{\varphi}= & \frac{1}{2} \Omega^{2} G^{\prime \prime}(x)-2 G^{\prime}(x) \Omega_{, x}-F^{\prime}(y) \Omega \Omega_{, y}-[G(x) \\
& \left.\times\left(\Omega \Omega_{, x x}-3 \Omega_{, x}^{2}\right)+F(y)\left(\Omega \Omega_{, y y}-3 \Omega_{, y}^{2}\right)\right] \\
R_{x}^{x}= & \frac{1}{2} \Omega^{2} G^{\prime \prime}(x)-2 G^{\prime}(x) \Omega \Omega_{, x}-F^{\prime}(y) \Omega \Omega_{, y}-[3 G(x) \\
& \left.\times\left(\Omega_{, x x}-\Omega_{, x}^{2}\right)+F(y)\left(\Omega \Omega_{, y y}-3 \Omega_{, y}^{2}\right)\right] \\
R_{y}^{y}= & \frac{1}{2} \Omega^{2} F^{\prime \prime}(y)-2 F^{\prime}(y) \Omega \Omega_{, y}-G^{\prime}(x) \Omega_{, x}-[G(x) \\
& \left.\times\left(\Omega_{, x x}-3 \Omega_{, x}^{2}\right)+3 F(y)\left(\Omega \Omega_{, y y}-\Omega_{, y}^{2}\right)\right] \\
R_{x y}=\frac{\Omega}{, x y} & \Omega
\end{aligned}
$$

The Einstein equations (51) then suggest

$$
\Omega=A(f+y)
$$

where $f_{0}=|x|$. Inputting this ansatz gives

$$
\frac{1}{2}\left(R_{\varphi}^{\varphi}-R_{x}^{x}\right)=G(x) A^{2} y(1+A|\mathbf{z}|) f_{, x x}=\frac{3 A}{2} \operatorname{sech}^{4} \frac{x}{A y}
$$

$$
\frac{1}{2}\left(R_{0}^{0}-R_{y}^{y}\right)=F(y) A^{2} y(1+A|\mathbf{z}|) f_{, y y}=O\left(A^{2}\right)
$$

$$
\begin{aligned}
\frac{1}{2}\left(R_{0}^{0}-\right. & \left.R_{\varphi}^{\varphi}\right)-q^{2} \Omega^{4} \\
= & A^{2}(y+f)\left[\left(1+3 m A|x|+2 q^{2} A^{2} x^{2}\right)\left(f-x f_{, x}\right)\right. \\
& -y f_{, y}+3 m A y\left(|x|-f+y f_{, y}\right)+q^{2} A^{2} \\
& \left.\times\left[\left(x^{2}-f^{2}\right)(f+3 y)-2 y^{3} f_{, y}\right]\right] \\
= & O\left(A^{2}\right) .
\end{aligned}
$$

Using [25], Eq. (54a) has solution

$$
f=A y\left[\ln \cosh \frac{x}{A y}+\frac{1}{4} \tanh ^{2} \frac{x}{A y}\right]
$$

note that while $f_{, x}=O(A), f_{, y}=\left(f-x f_{, x}\right) / y=O\left(A^{2}\right)$, and the Einstein equations are satisfied to leading order in $A$.

Therefore, the topological kink solution smooths out the shell-like singularity of the infinitesimal domain wall in much the same way as the topological local vortex solution smooths out the delta-function singularity in the AFV and other metrics. 


\section{NUCLEATION OF BLACK HOLES ON WALLS}

Both cosmic strings and domain walls are objects with a tension that tends to make them unstable to snapping or forming holes on them. In the absence of gravity, they may be protected against such instabilities by the topology of the field configuration that gives rise to them. However, it has been known for some time now that even topologically stable vortices are unstable to snapping by forming a pair of black holes at their end points [7]. This is a quantum tunneling process, mediated by an instanton obtained from the Euclidean continuation of the same $C$ metric as in Eq. (1).

An investigation of a related instability for domain walls was initiated in [27]. It was found that domain walls could nucleate black holes at a finite distance from them. That is, a spherical domain wall that is accelerating in an otherwise empty Minkowski space [such as we have described in Eqs. (11)-(13)] may tunnel to a configuration where it encloses a black hole. Actually, given the double-sided nature of the wall, it encloses two black holes, one on each side of the wall. It should be noted that the description of such tunneling process is not without its qualms, since given the compact nature of the Euclidean solutions involved, the wall (and the entire universe with it), must be annihilated before giving way to the configuration where the wall encloses the black hole. $^{3}$

This instability of the domain wall, however, is not quite the analogue of the snapping string. Instead, we shall describe now how black holes can form on a domain wall (even on a topologically stable one)_an instability that might be dubbed the hole-punch mechanism.

The final state will be precisely the one we have been describing in the previous sections: an accelerating spherical domain wall with a pair of black holes grafted at antipodal points on the wall. The black holes do not swallow the brane, hence the holes do not grow. Again, the transition to this configuration involves a quantum fluctuation where the initial domain wall is annihilated, to be recreated back with the black holes on it. The probability for this to happen is given, in first approximation, by $\exp \left[-\left(I-I_{0}\right)\right]$, where the Euclidean action $I_{0}$ of the initial configuration (the wall without a black hole) is subtracted from the Euclidean action $I$ of the final state with the black holes on the wall (we are assuming noboundary conditions for the wave functions of the corresponding universes). Alternatively, $\exp \left[-\left(I-I_{0}\right)\right]$ can be viewed as the ratio of the probabilities to nucleate a domain wall with and without black holes riding on it.

Let us proceed to construct the wall-black-hole instanton. After continuation $t \rightarrow i \tau$, in order to get the correct signature we restrict the range of $y$ to $-\xi_{3} \leqslant y \leqslant-\xi_{2}$. The end points of this interval are spherical bolts (two-dimensional fixed point sets of $\partial_{\tau}$ ) and in order to avoid the appearance of conical singularities at them the Euclidean time coordinate has to be periodically identified, $\tau \sim \tau+\beta$, in such a way that

\footnotetext{
${ }^{3}$ This is familiar also from processes of black hole nucleation in an inflating universe [29].
}

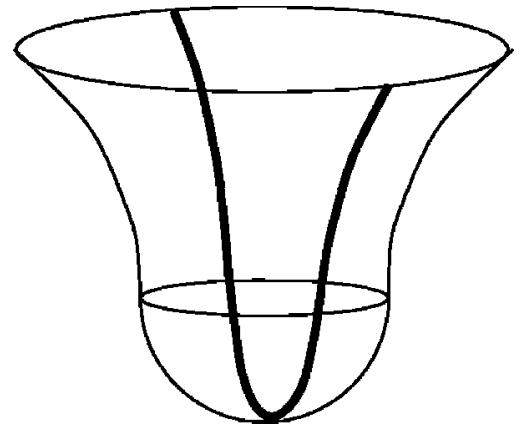

FIG. 4. The instanton process for nucleation of a domain wall with black holes on it. The lower half-sphere is half the Euclidean instanton, which then tunnels to real time and the hyperbolic expansion of the wall. The black holes are created as a particleantiparticle pair. An identification of this geometry to a copy of itself along the boundary $x=0$ must be performed, as in Fig. 2 .

$$
\beta=\frac{4 \pi}{G^{\prime}\left(\xi_{3}\right)}=\frac{4 \pi}{\left|G^{\prime}\left(\xi_{2}\right)\right|} .
$$

The second equality cannot be fulfilled unless we appropriately restrict the parameters of the solution. Specifically, this equation requires $\xi_{1}-\xi_{2}-\xi_{3}+\xi_{4}=0$. The physical interpretation of this constraint is simple, and corresponds to a condition of thermal equilibrium: the temperature of the black hole must be the same as the one induced by the acceleration horizon. A neutral black horizon is smaller, hence hotter, than an acceleration horizon, and as a consequence the above equation has no solutions if $q=0$. If charge is added to the black hole, its temperature can be lowered and then tuned to match the acceleration temperature. One could also consider extremal black holes, for which $\xi_{1}=\xi_{2}$ so that the black hole horizon does not restrict $\beta$. This can be accommodated easily in what follows, and does not lead to qualitatively new results.

The hole-punch instanton thus constructed (see Fig. 4) is completely non-singular (save for the smoothable singularity at the location of the wall), and it is also compact, which considerably facilitates the calculation of its action. The latter can be computed directly by plugging the explicit form of the solution into

$$
I=-\frac{1}{16 \pi} \int d^{4} x \sqrt{g}\left(R-F^{2}\right)+\sigma \int d^{3} x \sqrt{h}
$$

$(G=1)$. This expression, in fact, can be further simplified on-shell by use of the equations of motion which allow one to eliminate, say, $R$ in favor of $F^{2}$ and the wall action. Alternatively, one can use the on-shell equation [28]

$$
I=-\frac{1}{4}\left(\mathcal{A}_{\mathrm{acc}}+\mathcal{A}_{\mathrm{bh}}\right)
$$

which gives the action in terms of the area of the horizons, which are all finite in the present solution. Both methods yield, of course, the same result, but the latter is perhaps 
slightly simpler. The area of the black hole horizon has already been computed in Eq. (15), and that of the acceleration horizon is, analogously,

$$
\mathcal{A}_{\mathrm{acc}}=2 \frac{\Delta \varphi}{A^{2}} \frac{\xi_{4}}{\xi_{3}\left(\xi_{3}-\xi_{4}\right)} .
$$

Collecting all terms, we obtain

$$
I=-\frac{1}{8 \pi \sigma^{2}} \frac{4 \xi_{4}}{\left|G^{\prime}\left(\xi_{4}\right)\right|}\left(\frac{1}{\xi_{3}\left(\xi_{3}-\xi_{4}\right)}+\frac{1}{\xi_{2}\left(\xi_{2}-\xi_{4}\right)}\right) .
$$

On the other hand, the action for a domain wall instanton (without black holes) is

$$
I_{0}=-\frac{1}{8 \pi \sigma^{2}}
$$

and, as we said, the rate for nucleating holes on the wall is $\sim \exp \left[-\left(I-I_{0}\right)\right]$. The resulting expression is not too illuminating, but it simplifies in the limit of small black holes, $M, Q \ll \sigma \propto 1 / A$,

$$
I=-\frac{1}{8 \pi \sigma^{2}}\left(1-8 \pi M \sigma+O\left(M^{2} \sigma^{2}\right)\right)
$$

(recall that the black holes are nearly extremal, so $M \simeq Q$ ). Hence,

$$
I-I_{0} \simeq \frac{M}{\sigma}
$$

which, we observe, is a positive quantity. Nucleation of black holes is exponentially suppressed, as was to be expected. It may be observed that, to this order, only $\mathcal{A}_{\text {acc }}$ contributes. The black hole entropy $\sim 4 \pi M^{2}$ contributes to enhance the nucleation rate, but enters only at the next order.

We have demonstrated how, in addition to the process of nucleation of black holes enclosed by a wall described in [27], black holes can nucleate on the wall. The question arises of which instability the domain wall is more likely to undergo. The two processes are actually rather different, so one has to specify which final states one is comparing. In [27] it was found that the action difference for nucleation of a neutral black hole inside the wall is

$$
I-I_{0}=\frac{11 \sqrt{3}}{36} \frac{M}{\sigma} \approx 0.53 \frac{M}{\sigma}
$$

which would be smaller than Eq. (63) and hence the process would appear to be less suppressed. However, there is a crucial difference: in the configuration where the black hole is enclosed by the wall, the mass of the black hole is fixed to be $M=(6 \sqrt{3} \pi \sigma)^{-1}$ and hence cannot be varied independently of the tension of the wall. In fact, the geometry is that of the Schwarzschild solution, with the wall sitting at a fixed radius $r=3 M$. In other words, domain walls can only nucleate inside them black holes of a certain (large) size. As a result, the process of [27] can only lead to the formation of very large black holes, and therefore will be heavily suppressed. In contrast, in the hole-punch process the black hole mass $M$ is a parameter that can be varied independently of $\sigma$. Therefore, Eq. (63) can be made arbitrarily small. ${ }^{4}$ We conclude that domain walls will preferentially nucleate small black holes on them, rather than large ones inside them.

One might still be interested in comparing the processes where the domain walls nucleate black holes with the same features-we take the black holes to be extremal or nearly extremal. To this effect, we should compare Eq. (63) to the nucleation rate of a (nearly) extremal charged black hole inside the wall. In that case, $M$ is again fixed in terms of $\sigma$, $M=Q=1 /(8 \pi \sigma)$, and $^{5}$

$$
I-I_{0}=\frac{3}{32 \pi \sigma^{2}}=\frac{3}{4} \frac{M}{\sigma},
$$

again smaller than Eq. (63). However, this result is exact, whereas corrections to Eq. (63) (which can be seen to lower its value) will be quite important since we are supposed to be taking $m A \simeq 2 \pi M \sigma=1 / 4$, not a small number. And, in any event, the hole-punch process can form nearly extremal black holes much smaller than this, which will be less suppressed.

\section{CONCLUSIONS}

In this paper we have considered the problem of having a black hole sitting on a topological domain wall, necessarily including the gravitational back reaction. A domain wall has a very strong effect on the spacetime surrounding it, causing a compactification of spatial sections. We started by deriving the metric for an infinitesimally thin domain wall bisecting a black hole, using the $C$ metric in a recently developed construction [16]. The global structure of this spacetime is the interior of two hyperboloids in a Lorentzian spacetime (see Fig. 2) with two accelerating black holes "glued" to these walls. If the horizons are identified, then the black holes are joined by a wormhole - this indeed happens if the black holes are nucleated as a pair. We have used thermodynamics to provide a definition for the mass of the black hole, which led us to conclude that it is entropically preferred to have the black hole on the wall, rather than away from it. We showed how one can smooth out the "singular" behavior of the zero thickness wall by using a core of a topological, and hence thick, domain wall. Meanwhile for extreme black holes, while the picture is qualitatively the same, if the wall is thick enough relative to the black hole (roughly bigger than the black hole size) the black hole will expel its flux, in the sense that the scalar field forming the wall will remain in its false vacuum, restored-symmetry state on the event horizon. This phenomenon might have consequences for any brane-world model in which such charged black holes are possible-

\footnotetext{
${ }^{4}$ The semiclassical approximation, however, will break down when the black hole mass reaches the Planck scale.

${ }^{5}$ Of all the situations considered in [27] we are only taking the process for which the action is smaller.
} 
recall that these black holes are charged under a gauge field that is not confined to the brane. If one tries to pull a small extremal black hole out of the brane, it will not experience the same elastic restoring force that non-extremal black holes suffer, so, apparently they might be able to slip off of the brane into the bulk.

The problem of black hole nucleation on the domain wall - the hole-punch process - is also analyzed and we conclude it is more probable than the black hole nucleation away from the wall considered in [27].

We want to conclude by discussing the possible extension of the results in this paper to one dimension higher, this is, to the scenario with a three-brane in five dimensions, which is pertinent for brane world models.

The first, and very serious, obstacle is the lack of an analogue of the $C$ metric solution in five dimensions. Even if it is fairly safe to assume, on physical grounds, that such a spacetime must exist, no explicit construction of it has been found. Hence, the five dimensional analogue of the wallblack hole metric remains unknown. In contrast, there should be no problem in studying a black hole intersected by a domain wall in five dimensions if the gravitational backreaction of the latter is ignored. Including the backreaction in a perturbative fashion might give some clues to the full solution. On the other hand, the phenomenon of flux expulsion appears to be essentially dimension independent.

The process of nucleation of black holes on walls presents some peculiarities in five dimensions. Again, the absence of an explicit analogue of the $C$ metric precludes a conclusive analysis. Nevertheless, in [30] an instanton was presented which mediated the nucleation of a domain wall in five dimensions, with a "black object" on it. On the brane, as well as on any surface at constant radius away from the brane, the four-geometry is that of the Nariai instanton $S^{2} \times S^{2}$ (a limiting case of the Euclidean Schwarzschild-de Sitter solution). Hence, the black object does not seem to be a black hole localized on the brane, but rather a black string extending throughout the bulk-note, however, that the authors of [30] argue otherwise. The size of the black objects nucleated via this instanton is, as was the case in [27], fixed by the wall tension. This is, the mass of the black object is not an independent parameter. In contrast, we have argued that, in the four dimensional setting, a domain wall can nucleate black holes of arbitrarily small size-which are preferred over larger black holes. It seems reasonable to assume that a similar process will be possible in five dimensions, and small black holes will be nucleated on the brane. Nevertheless, notice that Euclidean regularity demanded that the black hole be endowed with charge with respect to a bulk gauge field. Such gauge fields are not always present in brane world models. The absence of an explicit wall-black hole solution in five dimensions leaves the door open to unexplored alternatives, but it might be that, if no such charges are allowed, then the only possible instanton for nucleation of walls with black objects on them, were that of [30].

\section{ACKNOWLEDGMENTS}

We would like to thank Christos Charmousis for discussions. This work has been supported by a Royal Society European Exchange Program grant. R.E. acknowledges partial support from UPV grant 063.310-EB187/98 and CICYT AEN 99-0315, R.G. is supported by the Royal Society, and C.S. is supported by Fundação Ciencia e Tecnologia BPD/ 22092/9.
[1] P. T. Chrusciel, " No hair theorems-Folklore, Conjectures, Results,” gr-qc/9402032.

[2] R. Bartnick and J. McKinnon, Phys. Rev. Lett. 61, 141 (1988); K. Lee, V. Nair, and E. Weinberg, ibid. 68, 1100 (1992); F. Dowker, R. Gregory, and J. Traschen, Phys. Rev. D 45, 2762 (1992).

[3] A. Achucarro, R. Gregory, and K. Kuijken, Phys. Rev. D 52, 5729 (1995).

[4] M. Aryal, L. Ford, and A. Vilenkin, Phys. Rev. D 34, 2263 (1986).

[5] W. Israel and K. A. Khan, Nuovo Cimento 33, 331 (1964).

[6] W. Kinnersley and M. Walker, Phys. Rev. D 2, 1359 (1970).

[7] R. Gregory and M. Hindmarsh, Phys. Rev. D 52, 5598 (1995);

R. Emparan, ibid. 52, 6976 (1995); D. Eardley, G. Horowitz, D. Kastor, and J. Traschen, Phys. Rev. Lett. 75, 3390 (1995); S. W. Hawking and S. F. Ross, ibid. 75, 3382 (1995); R. Emparan, ibid. 75, 3386 (1995).

[8] V. A. Rubakov and M. E. Shaposhnikov, Phys. Lett. 125B, 136 (1983); K. Akama, in Gauge Theory and Gravitation, Proceedings of the International Symposium, Nara, Japan, 1982, edited by K. Kikkawa, N. Nakanishi, and H. Nariai (SpringerVerlag, Berlin, 1983).

[9] P. Horava and E. Witten, Nucl. Phys. B475, 94 (1996); A.
Lukas, B. A. Ovrut, K. S. Stelle, and D. Waldram, Phys. Rev. D 59, 086001 (1999); A. Lukas, B. A. Ovrut, and D. Waldram, ibid. 60, 086001 (1999).

[10] N. Arkani-Hamed, S. Dimopoulos, and G. Dvali, Phys. Lett. B 429, 263 (1998); I. Antoniadis, N. Arkani-Hamed, S. Dimopoulos, and G. Dvali, ibid. 436, 257 (1998).

[11] M. Visser, Phys. Lett. 159B, 22 (1985); E. J. Squires, ibid. 167B, 286 (1986).

[12] L. Randall and R. Sundrum, Phys. Rev. Lett. 83, 3370 (1999); 83, 4690 (1999).

[13] A. G. Cohen and D. B. Kaplan, Phys. Lett. B 470, 52 (1999); R. Gregory, Phys. Rev. Lett. 84, 2564 (2000); T. Gherghetta and M. Shaposhnikov, ibid. 85, 240 (2000); M. Gogberashvili and P. Midodashvili, "Brane universe in six dimensions," hep-ph/0005298; I. Olasagasti and A. Vilenkin, Phys. Rev. D 62, 044014 (2000).

[14] J. Garriga and T. Tanaka, Phys. Rev. Lett. 84, 2778 (2000).

[15] A. Chamblin, S. W. Hawking, and H. S. Reall, Phys. Rev. D 61, 065007 (2000).

[16] R. Emparan, G. T. Horowitz, and R. C. Myers, J. High Energy Phys. 01, 007 (2000); 01, 021 (2000).

[17] M. Gremm, Phys. Lett. B 478, 434 (2000).

[18] Y. Morisawa, R. Yamazaki, D. Ida, A. Ishibashi, and K. Na- 
kao, Phys. Rev. D 62, 084022 (2000).

[19] J. Ipser and P. Sikivie, Phys. Rev. D 30, 712 (1984); A. Vilenkin, Phys. Lett. 133B, 177 (1983).

[20] G. W. Gibbons, Nucl. Phys. B394, 3 (1993); M. Cvetic, S. Griffies, and H. Soleng, Phys. Rev. Lett. 71, 670 (1993); Phys. Rev. D 48, 2613 (1993).

[21] A. Chamblin, J. Ashbourn-Chamblin, R. Emparan, and A. Sornborger, Phys. Rev. Lett. 80, 4378 (1998); Phys. Rev. D 58, 124014 (1998).

[22] A. Chamblin, R. Emparan, and G. W. Gibbons, Phys. Rev. D 58, 084009 (1998).

[23] F. Bonjour and R. Gregory, Phys. Rev. Lett. 81, 5034 (1998); F. Bonjour, R. Emparan, and R. Gregory, Phys. Rev. D 59,
084022 (1999).

[24] R. Basu and A. Vilenkin, Phys. Rev. D 46, 2345 (1992); 50, 7150 (1994).

[25] F. Bonjour, C. Charmousis, and R. Gregory, Class. Quantum Grav. 16, 2427 (1999).

[26] R. Emparan, G. T. Horowitz, and R. C. Myers, Phys. Rev. Lett. 85, 499 (2000).

[27] R. R. Caldwell, A. Chamblin, and G. W. Gibbons, Phys. Rev. D 53, 7103 (1996).

[28] S. W. Hawking and G. T. Horowitz, Class. Quantum Grav. 13, 1487 (1996).

[29] R. B. Mann and S. F. Ross, Phys. Rev. D 52, 2254 (1995).

[30] J. Garriga and M. Sasaki, Phys. Rev. D 62, 043523 (2000). 